\title{
Vibrational Dynamics of Crystalline 4-Phenylbenzaldehyde from INS Spectra and Periodic DFT Calculations
}

\author{
Mariela M. Nolasco ${ }^{1, *(\mathbb{D}}$, Catarina F. Araujo ${ }^{1}$, Pedro D. Vaz $^{2} \mathbb{D}^{\mathbb{D}}$, Ana M. Amado ${ }^{3} \mathbb{D}^{\mathbb{D}}$ and \\ Paulo Ribeiro-Claro ${ }^{1}$ D \\ 1 CICECO, Departamento de Química, Universidade de Aveiro, P-3810-193 Aveiro, Portugal; \\ catarina.araujo@ua.pt (C.F.A.); prc@ua.pt (P.R.-C.) \\ 2 Champalimaud Foundation, Champalimaud Centre for the Unknown, 1400-038 Lisboa, Portugal; \\ pedro.vaz@fundacaochampalimaud.pt \\ 3 Química-Física Molecular, Departamento de Química, FCTUC, Universidade de Coimbra, \\ P-3004-535 Coimbra, Portugal; amado.am@gmail.com \\ * Correspondence: mnolasco@ua.pt; Tel.: +351-234-370-360
}

Received: 24 February 2020; Accepted: 16 March 2020; Published: 18 March 2020

\begin{abstract}
The present work emphasizes the value of periodic density functional theory (DFT) calculations in the assessment of the vibrational spectra of molecular crystals. Periodic calculations provide a nearly one-to-one match between the calculated and observed bands in the inelastic neutron scattering (INS) spectrum of crystalline 4-phenylbenzaldehyde, thus validating their assignment and correcting previous reports based on single molecule calculations. The calculations allow the unambiguous assignment of the phenyl torsional mode at ca. $118-128 \mathrm{~cm}^{-1}$, from which a phenyl torsional barrier of ca. $4000 \mathrm{~cm}^{-1}$ is derived, and the identification of the collective mode involving the antitranslational motion of $\mathrm{CH} \cdots \mathrm{O}$ bonded pairs, a hallmark vibrational mode of systems where $\mathrm{C}-\mathrm{H} \cdots \mathrm{O}$ contacts are an important feature.
\end{abstract}

Keywords: inelastic neutron scattering; density functional theory; vibrational assignment; molecular crystal; torsional potential; $\mathrm{C}-\mathrm{H} \cdots \mathrm{O}$ hydrogen bonds

\section{Introduction}

The use of periodic density functional calculations (periodic DFT) to address the vibrational spectra of molecular crystals is becoming increasingly popular [1-12]. In fact, the periodic DFT approach, although originally developed to deal with extended inorganic systems, was found to be highly efficient in predicting the vibrational spectra of molecular crystals [13]. Periodic methods-available through programs such as VASP [14], CRYSTAL [15,16] and CASTEP [17] —are often used to assist vibrational assignments of infrared and inelastic neutron scattering (INS) spectra of molecular crystals $[1,2,6-9,18]$. Vibrational spectroscopy with neutrons provides information not accessible from the optical techniques (IR and Raman), due to the absence of selection rules. The relationship between periodic DFT calculations and INS spectroscopy is somewhat synergistic. The intensity of INS bands is proportional to the relative atomic displacements in normal modes [19], which, in turn, are a straightforward result of the computational approach to vibrational frequencies. The ability of periodic calculations to predict INS intensities with high accuracy leads to mostly unambiguous assignments of vibrational modes and, additionally, provides the means to test the validity of the molecular model used $[6,20]$.

The crystal structure of 4-phenylbenzaldehyde has been addressed in a comprehensive crystallographic, spectroscopic and computational study of $\mathrm{C}-\mathrm{H} \cdots \mathrm{O}$ hydrogen bonds [21]. More recently, the complete assignment of infrared and Raman spectra has been proposed based on discrete 
DFT calculations [22]. These works try to describe the vibrational spectra of the crystal through discrete calculations, either by choosing the simpler and most common "isolated molecule" approach [22] or by using several "dimer associations" to represent the possible interactions in the crystal [21]. Hence, 4-phenylbenzaldehyde is a suitable subject to test the "computational spectroscopy" approach to molecular crystals using periodic calculations. By combining periodic DFT calculations with INS spectra it is possible to validate assignments and assess vibrational dynamics of 4-phenylbenzaldehyde in the crystal.

\section{Results and Discussion}

\subsection{Molecular Geometry and Intermolecular Interactions}

Figure 1 presents a fragment of the crystal structure of 4-phenylbenzaldehyde, along with the numbering scheme adopted in this work. The crystal structure (monoclinic, space group P21/a, $\mathrm{Z}=4$ ) [21] is built from two types of dimers: the $\mathrm{C}-\mathrm{H} \cdots \mathrm{O}$ bonded dimers (molecules labeled A) coexist with pi-stacking dimers (molecules labeled B), as shown in Figure 1. All molecules are involved in both types of dimers, but they have slightly different secondary neighboring contacts.

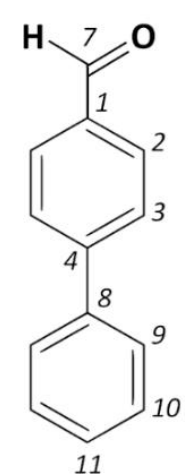

11

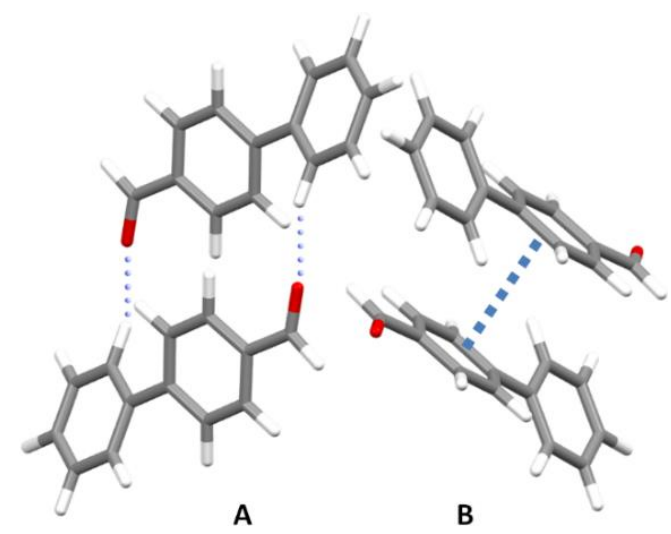

Figure 1. Representation of 4-phenylbenzaldehyde, with the atom labeling used throughout the text (left) and fragment of the crystal structure, evidencing the two types of dimer present in the crystal (right) [21].

Table 1 compares some selected geometrical parameters obtained from X-ray [21], periodic calculations (CASTEP, see experimental details) and discrete single molecule calculations (G09, see experimental details). The "single molecule" model was chosen to perform the discrete calculations as it remains, despite its known limitations, a popular approach. The "dimer" model is not a straightforward option, due to the presence of different intermolecular contacts with identical strength $(\mathrm{C}-\mathrm{H} \cdots \mathrm{O}$ and pi-stacking, dimers A and B in Figure 1). The alternative use of a "cluster" of molecules poses two main problems: the large number of molecules required to mimic the periodic crystal motif and the erratic behavior of the outer shell of the cluster (bound by weak intermolecular forces). In the past, a method based on the sum of pairwise contributions has been used as a less computationally demanding alternative to the cluster model [23-25] but with limited advantages.

There is a good agreement between experimental and calculated crystal structures (X-ray vs. CASTEP). The root mean square deviation between Cartesian coordinates of all atoms, excluding hydrogen atoms, is less than 5\%, and RMS deviation in bond lengths, excluding C-H bonds, is less than $2 \%$. Nevertheless, CASTEP geometrical parameters present some well-known limitations of PBE calculations. The $\mathrm{C}=\mathrm{O}$ bond length is clearly overestimated-leading to the underestimation of the $\mathrm{C}=\mathrm{O}$ stretching mode, already discussed elsewhere $[2,8]$ —and the pi-stacking distance evidences the expected bias to large values: this distance would deviate to physically meaningless values if not constrained by cell dimensions [2]. 
Table 1. Selected geometrical parameters of 4-phenylbenzaldehyde (molecules in dimers A and B).

\begin{tabular}{cccccc}
\hline & X-ray (A) & X-ray (B) & CASTEP(A) & CASTEP(B) & G09 \\
\hline Bond length/pm & & & & & \\
C7=O & 118.1 & 118.7 & 122.7 & 122.8 & 120.6 \\
C4-C8 & 149.2 & 145.5 & 147.8 & 147.8 & 147.7 \\
C1-C7 & 148.7 & 148.4 & 146.8 & 146.8 & 147.4 \\
Dihedral anglep & & & & & \\
C2C1-C7O & -1.95 & -2.06 & -2.16 & -2.26 & -0.10 \\
C3C4-C8C9 & 31.9 & 31.6 & 32.6 & 31.0 & 38.9 \\
Distance/pm & & & & & \\
C9(H) -.O & 341 & 361 & 333.7 & 348.1 & - \\
$\pi \ldots \pi\left(\mathrm{C} 1 \cdots \mathrm{C}^{\prime}\right)^{1}$ & 414.7 & 411.1 & 418.5 & 418.1 & - \\
\hline
\end{tabular}

The geometry of the single molecule obtained from discrete calculations (G09) at the PBE1PBE/6-311G $(\mathrm{d}, \mathrm{p})$ level (Table S1, Supplementary Materials) is in good agreement with the one recently reported at the B3LYP/6-311++G(d,p) level [22] and does not present large deviations from the CASTEP geometry. Table 1 also evidences the effect of crystal packing on the dihedral angles of aldehyde and phenyl groups. For the isolated molecule, the $\mathrm{CHO}$ group is predicted to be nearly coplanar with the ring, while the non-planarity between the two rings is heightened relative to the packed molecules.

\subsection{Calculated vs. Experimental Spectra}

Figure 2, top line, presents the experimental INS spectrum of 4-phenylbenzaldehyde (TOSCA), in the $25-1800 \mathrm{~cm}^{-1}$ range. Figure 2, middle and bottom lines, show the simulated INS spectra obtained from periodic (CASTEP) and discrete (G09) calculations, respectively.

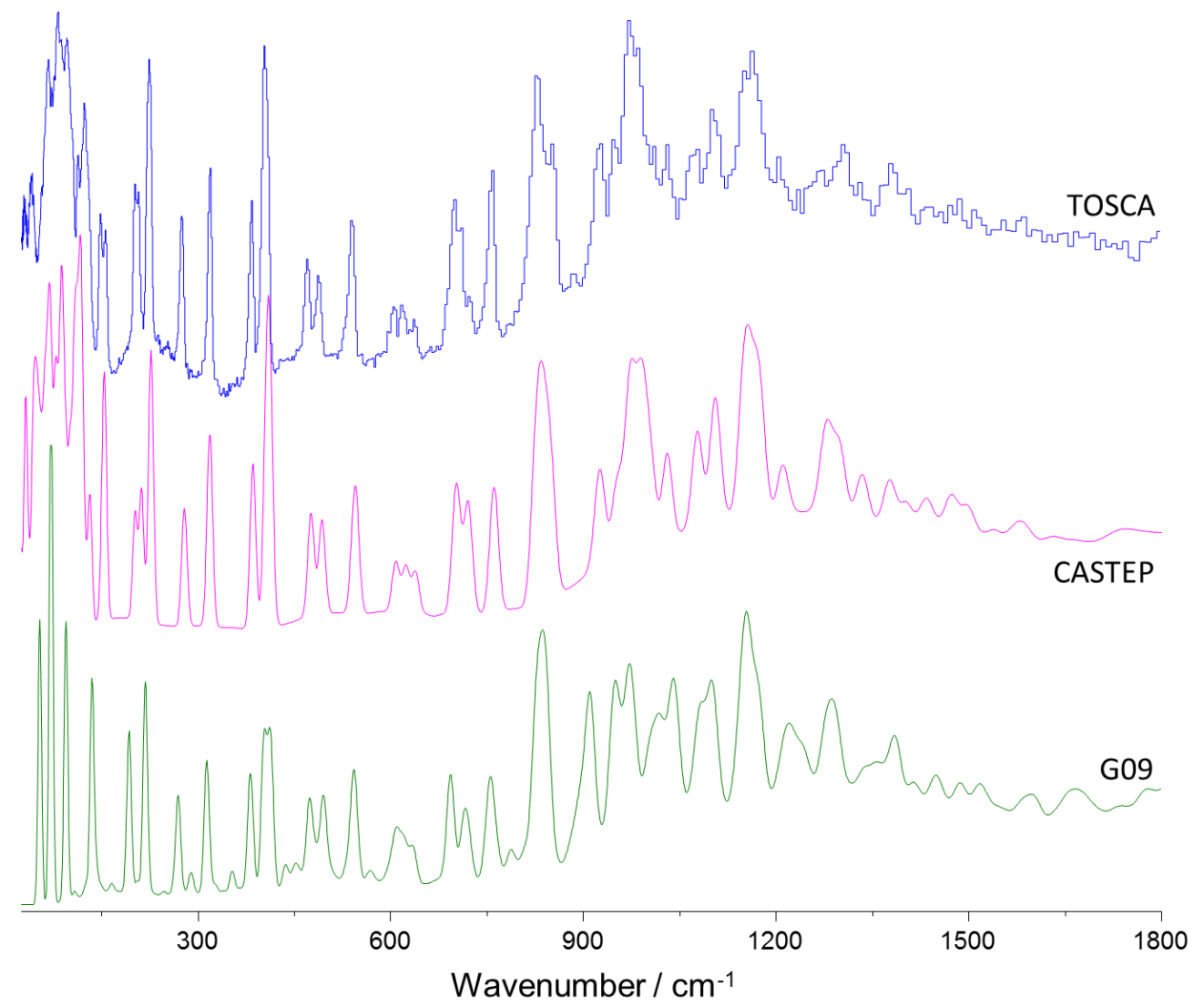

Figure 2. The inelastic neutron scattering (INS) spectra of 4-phenylbenzadhyde in the $25-1800 \mathrm{~cm}^{-1}$ range: experimental (top), simulated from periodic calculations (middle) and from single molecule discrete calculations (bottom). 
The immediate impression from Figure 2 is the excellent agreement between the experimental spectrum and the one estimated from periodic calculations, with a remarkable nearly one-to-one correspondence between the bands, in both their positions and intensities. As for the spectrum simulated using the single molecule approach (Figure 2, bottom), the correlation was quite satisfactory for some regions, but with clear overall limitations. For instance, there were a few "extra" bands spread in the simulated spectrum, without correspondence in the experimental spectrum, and the description of the region $900-1200 \mathrm{~cm}^{-1}$ was poor. Additionally, of course, the calculated spectrum for a single molecule was meaningless in the region where the contributions from collective modes were expected to prevail (ca. $<200 \mathrm{~cm}^{-1}$ ).

As stated above, the excellent description of the INS spectrum obtained from periodic calculations provided a straightforward validation of the model and allowed a trustful assignment of vibrational modes of crystalline 4-phenylbenzaldehyde. The full assignment of the INS bands based on the periodic calculations is presented in Table 2. The vibrational assignment of IR and Raman spectra of 4-phenylbenzaldehyde has been performed recently, based on discrete calculations at the B3LYP/6-311++G(d,p) level [22]. Although there is a general concordance between assignments, the INS spectrum allows the clarification of some spectral features, as discussed below.

Table 2. Observed (TOSCA) and calculated (CASTEP) INS wavenumbers of 4-phenylbenzaldehyde.

\begin{tabular}{|c|c|c|}
\hline CASTEP 1 & Observed & Approx. Description \\
\hline 3104 & 3030 & $v \mathrm{C}-\mathrm{H}$ \\
\hline 1379 & 1384 & $\beta C 7-\mathrm{H}$ \\
\hline 1298 & 1312 & $\beta C-H$ \\
\hline 1170 & 1164 & $\beta C-H$ \\
\hline 1106 & 1108 & $\beta \mathrm{C}-\mathrm{H}$ \\
\hline 1077 & 1075 & $\beta C-H$ \\
\hline 1030 & 1032 & $\beta C-H$ \\
\hline 994 & 994 & $\gamma \mathrm{C} 7-\mathrm{H}$ \\
\hline 975 & 973 & $\gamma \mathrm{C}-\mathrm{H}$ \\
\hline 953 & 951 & $\gamma \mathrm{C}-\mathrm{H}$ \\
\hline 926 & 929 & $\gamma \mathrm{C}-\mathrm{H}$ \\
\hline 845 & 855 & $\gamma \mathrm{C}-\mathrm{H}$ \\
\hline 833 & 832 & $v C-C$ \\
\hline 762 & 762 & $\gamma \mathrm{C}-\mathrm{H}$ \\
\hline 720 & 715 & Sring \\
\hline 702 & 702 & Sring \\
\hline 638 & 641 & $\alpha$ ring \\
\hline 622 & 623 & $\beta C=\mathrm{O}$ \\
\hline 609 & 610 & $\alpha$ ring \\
\hline 545 & 545 & dring \\
\hline 493 & 493 & $\alpha$ ring \\
\hline 476 & 475 & Sring \\
\hline 408 & 408 & Sring \\
\hline 385 & 388 & $\beta C 8-C 4$ \\
\hline 317 & 324 & $\gamma \mathrm{C} 8-\mathrm{C} 4$ \\
\hline 278 & 281 & $\alpha$ ring \\
\hline 225 & 229 & $\gamma \mathrm{C}-\mathrm{CHO}$ \\
\hline $210-201$ & $212-207$ & $\beta \mathrm{C}-\mathrm{CHO}$ \\
\hline 153 & $152-162$ & $\tau-\mathrm{CHO}$ \\
\hline 130 & 136 & $\beta C 4-C 8$ \\
\hline $109-117$ & $118-128$ & $\tau-\mathrm{C}_{6} \mathrm{H}_{5}$ \\
\hline 89 & 101 & Libration (Ia) \\
\hline 67 & 86 & Librations (Ic, Ib) \\
\hline 48 & 70 & Translation $(b, c)$ \\
\hline 37 & 45 & Translation(a) \\
\hline
\end{tabular}

${ }^{1}$ Maxima in the INS simulated spectrum. $\nu, \alpha, \beta, \gamma$ and $\tau$ stand for stretching, in-plane deformation, out-of-plane deformation and torsion modes, respectively. Librations around the molecular axes of inertia, translations along cell axes. 
The 4-phenylbenzaldehyde crystal presents 192 atoms in the crystallographic unit cell (24 atoms per isolated molecule) [21], from which 573 optical modes are predicted. These include $8 \times 66$ normal vibrational modes of the eight molecules $(132 \mathrm{Ag}+132 \mathrm{Au}+132 \mathrm{Bg}+132 \mathrm{Bu})$ and 45 external modes describing translations and rotations (21 translational modes plus 24 librational modes, $12 \mathrm{Ag}+11 \mathrm{Au}+$ $11 \mathrm{Bg}+11 \mathrm{Bu})$.

The 66 normal modes of vibration of each individual molecule include 60 related with the two phenyl rings. The "approximate description" of the normal modes in Table 2-obtained from the visualization of atomic displacements-is in accordance with the conventional notation for substituted aromatic rings: 23 stretching modes (labeled $v$ ), 18 in-plane deformation modes (labeled $\alpha$ ring and $\beta)$ and 18 out-of-plane deformation modes (labeled $\delta$ ring and $\gamma$ ). In addition, there is one torsional inter-ring mode, $\left(\tau-\mathrm{C}_{6} \mathrm{H}_{5}\right)$ and six modes related to the aldehyde group $(\nu C 7=\mathrm{O}, v C 7-\mathrm{H}, \beta C 7=\mathrm{O}$, $\beta C 7-\mathrm{H}, \gamma \mathrm{C} 7=\mathrm{O}$ and $\gamma \mathrm{C} 7-\mathrm{H}$, one of which is in fact the torsional mode, $\tau-\mathrm{CHO})$. This notation keeps the traditional separation between the "in-plane" and "out-of-plane" ring modes and assumes no significant interaction between internal and external modes. This latter assumption is expected to hold for systems with weak intermolecular binding [4], as it is the case of 4-phenylbenzaldehyde. However, the same is not true for the separation between "in-plane" and "out-of-plane" modes. Since the two aromatic rings are not coplanar, there is no molecular symmetry plane and the coupling between oscillators leads to normal modes of a hybrid nature. Figure 3 illustrates the case for the mode at ca. $832 \mathrm{~cm}^{-1}$. This mode is assigned to the $\mathrm{VC}-\mathrm{C}$ stretching mode of the benzaldehyde ring (carbon and hydrogen atoms move in the same direction), but the contribution from the out-of-plane $\gamma \mathrm{CH}$ modes is evident in the phenyl substituent ring (carbon and hydrogen atoms move in opposite directions).

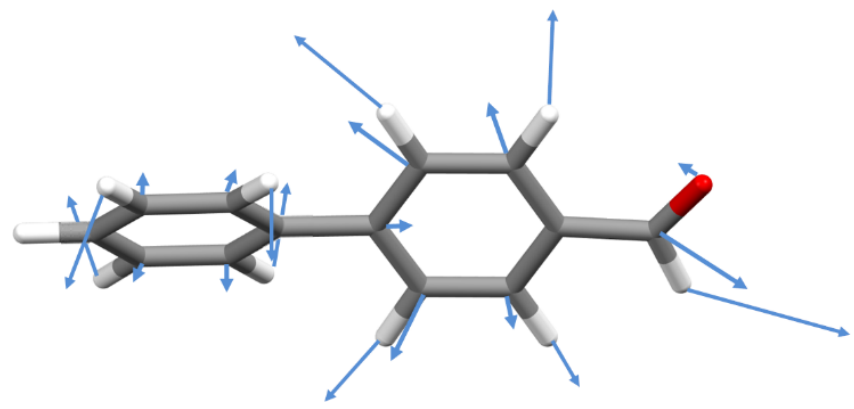

Figure 3. Atomic displacements of the normal mode at ca. $832 \mathrm{~cm}^{-1}$, assigned to a $v C-C$ stretching mode of the benzaldehyde ring.

The assignments in Table 2 can be compared with the ones recently reported [22]. The out-of-plane deformation mode of the aldehyde $\mathrm{CH}(\gamma \mathrm{C} 7-\mathrm{H})$ was clearly assigned to the intense INS band at $990 \mathrm{~cm}^{-1}$, while the band at ca. $1030 \mathrm{~cm}^{-1}$ was ascribed to the ring $\mathrm{CH}$ bending modes $(\beta \mathrm{C}-\mathrm{H})$. The mode at $832 \mathrm{~cm}^{-1}$ was better described as ring stretching $(\vee C C)$, despite its mixed nature, as described above. The contribution of ring deformation to the $623 \mathrm{~cm}^{-1}$ mode was high, but not more important than the $\mathrm{C} 1-\mathrm{C} 7=\mathrm{O}$ bond angle deformation, and thus the band was assigned to $\beta C=\mathrm{O}$. This bending mode has been misassigned to the band at $475 \mathrm{~cm}^{-1}$ [22], which is, in fact, an out-of-plane deformation of

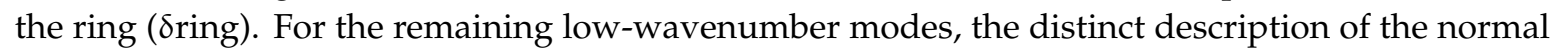
modes may arise from their complex mixture, which hampers the identification of the most relevant contribution in each case. This is an acknowledged limitation of the "approximate description" of the normal modes, chosen in favor of simplicity, as discussed elsewhere [2]. The exception is the inter-ring torsional mode (labeled phenyl torsion, $\tau-\mathrm{C}_{6} \mathrm{H}_{5}$ ), which is unambiguously found to occur at much higher energy (ca. 109-115 $\mathrm{cm}^{-1}$ ) than predicted from discrete calculations. 


\subsection{Large Amplitude, Low Wavenumber Motions}

One of the major advantages of INS spectroscopy is to afford rich information in the low wavenumber region, providing a glimpse into the molecular dynamics of the systems. Both large amplitude molecular (internal) modes and crystal lattice collective (external) modes present a strong intensity in the INS spectrum, mainly due to the large displacements of hydrogen atoms. Figure 4 compares the Raman, IR and INS spectra o crystalline 4-phenylbenzaldehyde in the region below $450 \mathrm{~cm}^{-1}$, evidencing the richness of the INS spectrum.

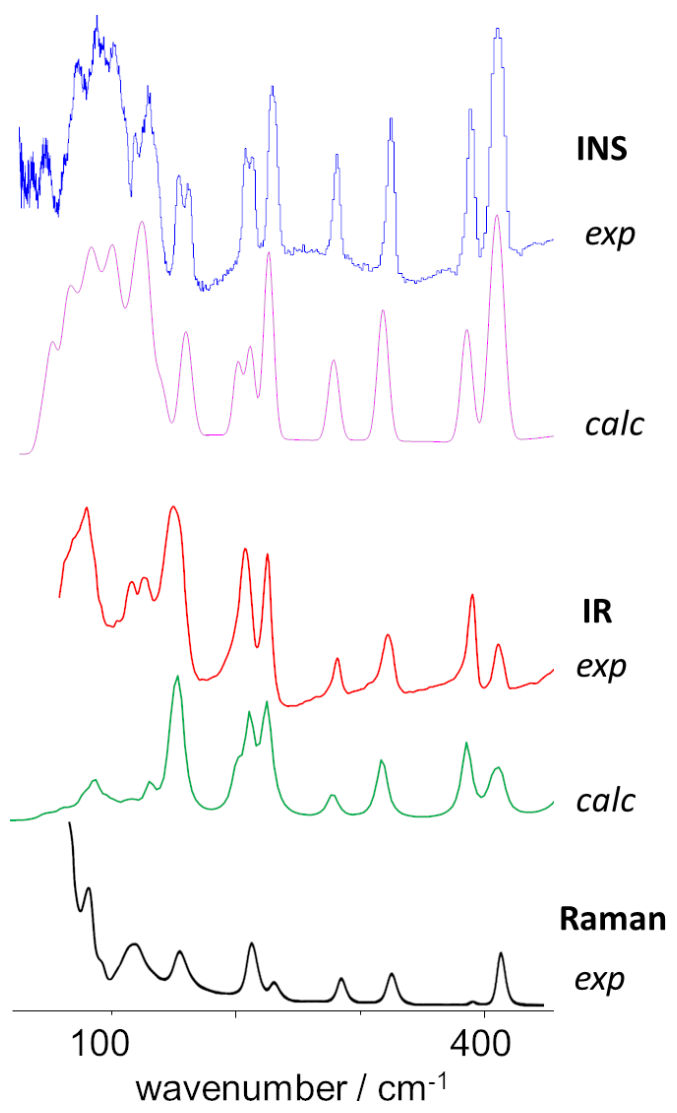

Figure 4. Vibrational spectra of 4-phenylbenzaldehyde in the spectral region below $450 \mathrm{~cm}^{-1}$. From top to bottom: INS spectra (experimental, calculated), far-infrared spectra (experimental, calculated) and Raman spectrum (experimental).

As stated above, there is little interaction between internal and external modes in 4-phenylbenzaldehyde, which is consistent with a crystal packing based on C-H...O and pi-stacking weak interactions. In this way, it is still plausible to describe the large amplitude/low wavenumber modes in terms of internal vibrations of the molecules vs. collective motions.

Among the most relevant internal low-wavenumber modes there are the torsional motions of the aldehyde and phenyl groups (-CHO and $\mathrm{C}_{6} \mathrm{H}_{5}-$, respectively). The torsion of the $-\mathrm{CHO}$ group is predicted to be at ca. $148-153 \mathrm{~cm}^{-1}$ from periodic calculations (CASTEP) and at ca. $139 \mathrm{~cm}^{-1}$ from discrete calculations (G09). This latter value compares with the one obtained from similar discrete calculations at the B3LYP level $\left(133 \mathrm{~cm}^{-1}\right.$ [22]).

As it can be seen in Figure 4, the calculated INS wavenumbers for-CHO torsion merge into a single band when assuming a reasonable linewidth. Nevertheless, the predicted band matches with the doublet found at ca. $152-162 \mathrm{~cm}^{-1}$, providing an unambiguous assignment. This value of the torsional frequency is above the value reported for the 4-fluorobenzaldehyde crystal $\left(120 \mathrm{~cm}^{-1}[4]\right)$, and suggests a somewhat stronger restriction of the $\mathrm{CHO}$ group dynamics in 4-phenylbenzaldehyde. 
The potential energy function for internal rotation is generally described by the sum of cosine terms,

$$
V(\theta)=\frac{1}{2} \sum_{n} V_{n}(1-\cos n \theta)
$$

from which the energy levels for torsional motion can be derived by solving the Hamiltonian for the internal rotor. More usefully, the barrier height can be estimated by fitting the energy level differences to the observed torsional transitions [26].

For the isolated molecule, the internal rotation potential obtained from discrete calculations (G09) can be described by the $\mathrm{V}_{2}$ component, $\mathrm{V}_{2}=3600 \pm 100 \mathrm{~cm}^{-1}(42.6 \pm 1.3 \mathrm{~kJ} / \mathrm{mol})$, consistent with the calculated torsional frequency of ca. $139 \mathrm{~cm}^{-1}$. This barrier height is in line with the one determined for benzaldehyde molecule from different quantum chemical calculations ( $\mathrm{ca} . \mathrm{V}_{2}=2700-2900 \mathrm{~cm}^{-1}$ ) [27]. For the crystalline state, the experimental value of $153 \mathrm{~cm}^{-1}$ can be obtained from a $\mathrm{V}_{2}$ barrier of nearly $4000 \pm 100 \mathrm{~cm}^{-1},(47.8 \pm 1.3 \mathrm{~kJ} / \mathrm{mol}$, see Figure 5, top panel). Even though the assumption of a pure $\mathrm{V}_{2}$ barrier in the crystal is a crude approximation, it suggests that the internal rotation dynamics of the $-\mathrm{CHO}$ group is mainly determined by intramolecular properties. The intermolecular interactions resulting from crystal packing represent a modest barrier height increase of ca. $5.2 \mathrm{~kJ} / \mathrm{mol}$, which is consistent with the presence of weak $\mathrm{CH} \cdots \mathrm{O}$ interactions [21].

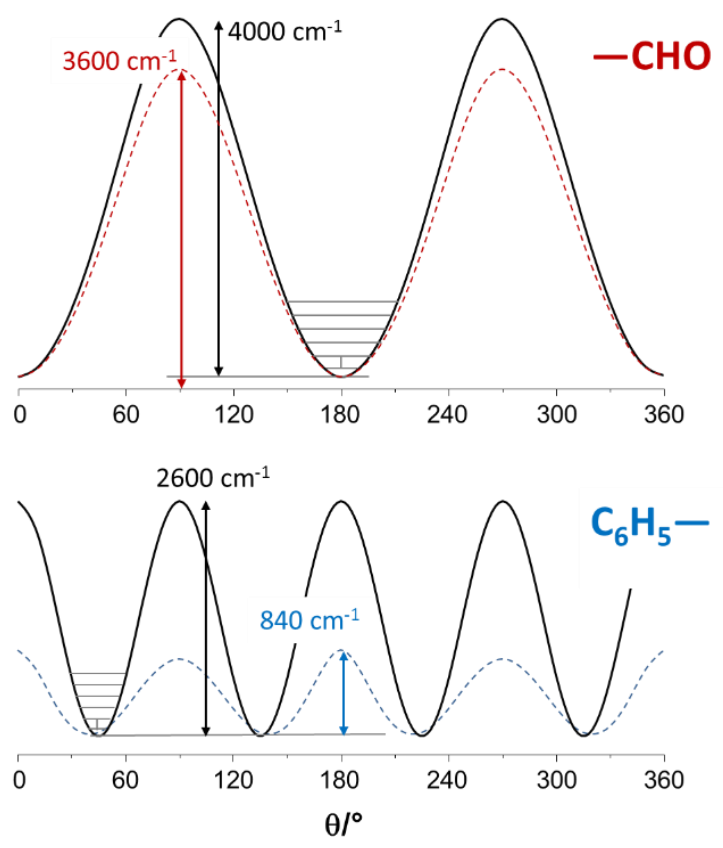

Figure 5. Potential curves for internal rotation of $-\mathrm{CHO}$ (top) and $\mathrm{C}_{6} \mathrm{H}_{5}-$ (bottom) groups in 4-phenylbenzaldehyde. Dashed lines from single molecule calculations (G09, energy vs. dihedral angle), solid lines from experimental wavenumber in the crystal (Barrier [26]). The G09 potential curve for phenyl internal rotation (blue dashed line) is described by the terms $V_{2}=-241 \mathrm{~cm}^{-1}, V_{4}=743 \mathrm{~cm}^{-1}$, $\mathrm{V}_{6}=126 \mathrm{~cm}^{-1}$ and $\mathrm{V}_{8}=51 \mathrm{~cm}^{-1}$.

As for the torsion of the phenyl substituent $\left(\mathrm{C}_{6} \mathrm{H}_{5}-\right)$, the effect of crystal packing is more pronounced. In fact, discrete calculations (G09) yield a torsional frequency value of only ca. $70 \mathrm{~cm}^{-1}$, while periodic calculations yield split torsional frequencies of $109-117 \mathrm{~cm}^{-1}$. Similar G09 calculations [22] lead to the assignment of phenyl torsion to a Raman band at ca. $65 \mathrm{~cm}^{-1}$. However, comparison between calculated and observed INS spectra in Figure 4 clearly suggests the assignment of the phenyl torsional mode in the crystal to the bands at ca. $118-128 \mathrm{~cm}^{-1}$, while the INS band at ca. $70 \mathrm{~cm}^{-1}$ is better ascribed to a collective "external" mode (Table 2). It should be mentioned that a phenyl torsion frequency of ca 
$116 \mathrm{~cm}^{-1}$ was recently identified in an ethylene terephthalate polymer (PET) from INS and CASTEP calculations [28].

The inter-ring torsional potential for the isolated molecule (G09) is dominated by a $\mathrm{V}_{4}$ component, with significant contributions from $\mathrm{V}_{2}, \mathrm{~V}_{6}$ and $\mathrm{V}_{8}$ (Figure 5, bottom panel). However, the calculated torsional frequency of $70 \mathrm{~cm}^{-1}$ can be obtained from a simplified approach, assuming a pure $\mathrm{V}_{4}$ term of ca. $860 \pm 30 \mathrm{~cm}^{-1}(10.3 \pm 0.3 \mathrm{~kJ} / \mathrm{mol})$. Within the same approximation, the observed frequency of $128 \mathrm{~cm}^{-1}$ for the crystal corresponds to a $V_{4}$ barrier of $2600 \pm 80 \mathrm{~cm}^{-1}$. The large difference indicates that, in this case, the barrier to internal rotation is mainly dominated by the intermolecular contacts-most probably the steric hindrance resulting from crystal packing, which is expected to be significant for a large group such as the phenyl group. The reports on the torsional barrier for the phenyl group are scarce, but there is a tendency for a pronounced increase from the gas phase to the condensed phase, as shown in Table 3.

Table 3. Comparison of $\mathrm{V}_{4}$ values for internal rotation of phenyl group derived from experiment.

\begin{tabular}{cccc}
\hline System & & $\mathbf{V}_{\mathbf{4}} / \mathbf{c m}^{\mathbf{- 1}}$ & Ref. \\
\hline 4-phenylbenzaldehyde & INS, crystal & $2600 \pm 80$ & This work \\
Biphenyl & Raman, crystal & $2650-200$ & {$[29]$} \\
Anisole & Raman, crystal & 4033 & {$[30]$} \\
Trans-Stilbene & Fluorescence, supersonic jet & 1550 & {$[31]$} \\
4-Methoxy-stilbene & Fluorescence, supersonic jet & 1430 & {$[32]$} \\
\hline
\end{tabular}

The 45 external modes of the crystallographic unit cell give rise to a limited number of bands in the low wavenumber region. Table 2 identifies the four main bands observed in this region, and Figure 6 presents the atomic displacements of some external modes with relevant contributions to these bands. Of course, there are several other combinations of translational and librational motions accounting for the final intensities of the four bands.
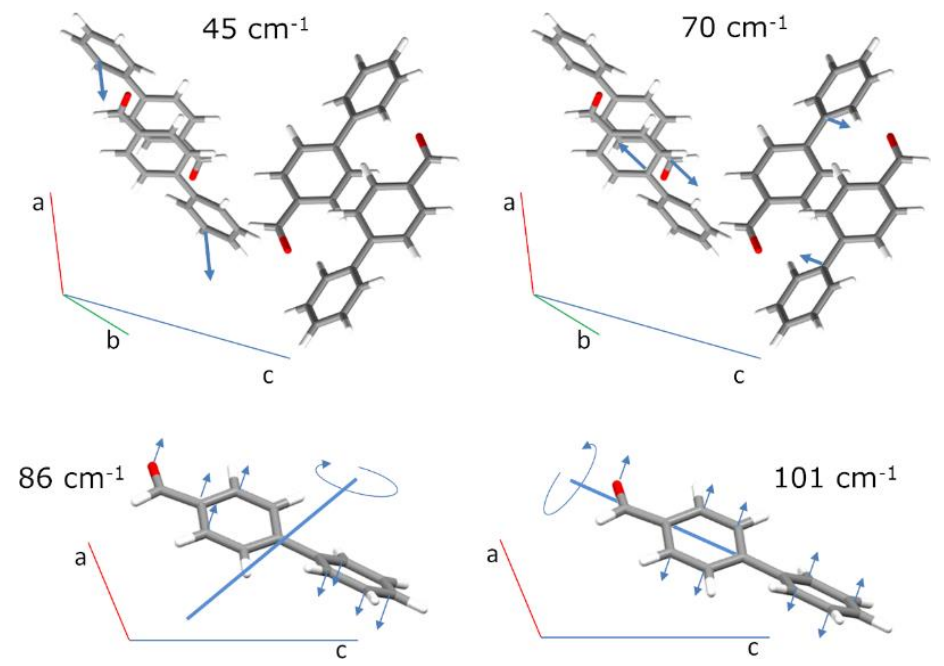

Figure 6. Schematic representation of atomic displacements for the external modes at $45 \mathrm{~cm}^{-1}, 70 \mathrm{~cm}^{-1}$ (translations), $86 \mathrm{~cm}^{-1}$ and $101 \mathrm{~cm}^{-1}$ (librations). For better readability, only the total displacement of the molecules is shown for translations and only the displacements of heavy atoms are shown for librations.

The translational mode at $70 \mathrm{~cm}^{-1}$ deserves particular discussion. As depicted in Figure 6, the full displacement of the molecules in this external mode results in the "slipping" between pi-stacked molecules, and the "antitranslational" motion of the $\mathrm{CH} \cdots \mathrm{O}$ bonded molecules. This antitranslational mode, which in fact represents the stretching vibration of the $\mathrm{H} \cdots \mathrm{O}$ intermolecular hydrogen 
bond $(v \mathrm{H} \cdots \mathrm{O})$, has been identified in other systems through INS spectroscopy [33-36]. In the chloroform-acetone complex this mode has been ascribed to the INS band at ca. $82 \mathrm{~cm}^{-1}$ [33]. More interestingly, cyclopentanone dimers-with a symmetric double $\mathrm{CH} \cdots \mathrm{O}$ contact, as found for 4-phenylbenzaldehyde dimer-present the anti-translational mode at $95 \mathrm{~cm}^{-1}$ [34]. Although the mode in 4-phenylbenzaldehyde is not a pure hydrogen bond stretching, as it also involves the ring slipping of pi-stacking dimers, it falls in the expected wavenumber range for the $\mathrm{CH} \cdots \mathrm{O}$ interaction.

\section{Materials and Methods}

4-Phenylbenzaldehyde was obtained commercially (Sigma-Aldrich, CAS number 459-57-4) and used as supplied.

The ATR-FTIR spectra of solid 4-phenylbenzaldehyde (Figure S1, Supplementary Materials) was measured on a Vertex 70 spectrometer using a Platinum ATR single reflection diamond accessory. For the far-IR spectrum $\left(50-600 \mathrm{~cm}^{-1}\right)$ a silicon solid-state beam-splitter and a Deuterated L-alanine doped TriGlycine Sulphate (DLaTGS) detector with a polyethylene window was used. The mid-IR spectrum (400-4000 $\mathrm{cm}^{-1}$ ) was recorded using a Ge on $\mathrm{KBr}$ substrate beam-splitter and a liquid nitrogen cooled wide band mercury cadmium telluride (MCT) detector. All spectra were the average of two counts of 128 scans each. A spectral resolution of $2 \mathrm{~cm}^{-1}$ was used.

The Raman spectrum was recorded on a Jobin-Yvon T64000 spectrometer, in the subtractive mode configuration, using a $514.5 \mathrm{~nm}$ argon ion laser line, a non-intensified charge coupled device (CCD) detector, with an integration time of $5 \mathrm{~s}$. The resolution was approximately $3 \mathrm{~cm}^{-1}$ and the estimated error in wavenumber was less than $1 \mathrm{~cm}^{-1}$.

INS spectrum: the inelastic neutron scattering experiment was performed with the TOSCA spectrometer, an indirect geometry time-of-flight spectrometer at the ISIS Neutron and Muon Source at the Rutherford Appleton Laboratory (Chilton, UK). The sample, with a total amount of ca. $2 \mathrm{~g}$, was packed inside a flat thin-walled aluminum can of $4.8 \mathrm{~cm}$ height and $4 \mathrm{~cm}$ width, with a path length of $2 \mathrm{~mm}$, which was mounted perpendicular to the beam, using a regular TOSCA centre-stick. The spectrum was collected below $15 \mathrm{~K}$, measured for the $16-8000 \mathrm{~cm}^{-1}$ energy-transfer range, and the resolution was $\Delta \mathrm{E} / \mathrm{E} \approx 1.5 \%$.

Quantum chemistry calculations: single molecule (discrete) calculations were performed with Gaussian 09 program (G09), version A.02 [37] using the PBE pure density functional combined with the 6-311G(d,p) basis set, as implemented in G09. Frequency calculations, subsequent to full geometry optimization, provide the infrared and Raman intensities and confirm the convergence to a true minimum (no imaginary frequencies). Potential energy functions for internal rotations have been obtained through the "scan" option (OPT = ModRedundant keyword) of G09, using the step size of $10^{\circ}$.

Periodic density functional theory (periodic-DFT) calculations were carried out using the plane-wave/pseudopotential method as implemented in the CASTEP code $[17,38]$. Exchange and correlation were approximated using the PBE functional [39]. The plane-wave cut-off energy was $830 \mathrm{eV}$. Brillouin zone sampling of electronic states was performed on the $8 \times 4 \times 4$ Monkhorst-Pack grid.

The equilibrium structure, an essential prerequisite for lattice dynamics calculations was obtained by LBFGS geometry optimization after which the residual forces were converged to zero within $0.005 \mathrm{eV} \cdot \mathrm{A}^{-1}$. The initial structure was taken from the reported crystal structure (CSD entry: WASLOS), and the cell parameters were kept constant during geometry optimization. This is important when using standard GGA functions, as their description of dispersion/van der Waals interactions is defective, leading to unrealistic cell dimensions. Phonon frequencies were obtained by the diagonalization of dynamical matrices computed using the density-functional perturbation theory. The eigenvectors (atomic displacements) for each normal mode that are part of the CASTEP output, enable visualization of the modes to aid assignments and are also all that is required to generate the INS spectrum using the program aClimax [40]. Program aClimax calculates INS intensities incorporating the instrumental 
bandwidth in a calculated spectrum that is easily compared with the experiment. It is emphasized that for all the calculated spectra shown the transition energies were not scaled.

Visualization of the atomic displacements of vibrational modes was performed using the Jmol program [41].

The determination of the potential barrier for a single simple internal rotor from torsional transitions has been performed with the program Barrier [26]. For an estimated error in torsional frequencies of less than $2 \mathrm{~cm}^{-1}$, the error in the barrier height was ca. $3 \%$.

\section{Conclusions}

The present work is a simple exercise that illustrates the capabilities of periodic DFT as an aid in the vibrational assignment of organic crystals. Despite being more resource intensive than its discrete counterparts, the effort of running periodic DFT calculations pays off by delivering accurate estimated spectra, which can be used as a direct guide for assignments. Even though 4-phenylbenzaldehyde individual units are held together by weak $\mathrm{C}-\mathrm{H} \cdots \mathrm{O}$ and pi-stacking interactions-therefore a prime candidate for the "cheaper" discrete model approach-a periodic description is necessary for reproducing the full vibrational features. An example of the latter is the phenyl torsion, whose frequency is severely underestimated by the single molecule approach, misleading its assignment. From the corrected torsional frequency, the torsional barrier value of $V_{4}=2600 \mathrm{~cm}^{-1}$ was obtained for the phenyl group, well above the value of $V_{4}=860 \mathrm{~cm}^{-1}$ predicted for the isolated molecule. This contrasts with the torsional barrier of the aldehyde group, - $\mathrm{CHO}$, which was clearly less affected by crystal packing $\left(4000 \mathrm{~cm}^{-1}\right.$ vs. $3600 \mathrm{~cm}^{-1}$, for the crystal and isolated molecule, respectively). In addition, periodic DFT is fundamental for understanding the low wavenumber region of INS spectra, offering a bird's-eye view of collective modes such as the antitranslational motion of $\mathrm{CH} \cdots \mathrm{O}$ bonded pairs, a hallmark vibrational mode of systems where $\mathrm{C}-\mathrm{H} \cdots \mathrm{O}$ contacts are an important feature.

Supplementary Materials: The following are available online, Figure S1: Mid Infrared spectrum of 4-phenylbenzaldehyde in the 400-4000 $\mathrm{cm}-1$ range (top), compared with the CASTEP calculated spectrum (bottom). Table S1: Coordinates of the optimized structure of 4-phenylbenzaldehyde (G09, PBEPBE/6-311G(d,p) keyword).

Author Contributions: Formal analysis, P.R.-C.; investigation, M.M.N. (INS, CASTEP), C.F.A. (G09), P.D.V. (INS), A.M.A. (Raman, Far-IR); writing-original draft preparation, M.M.N.; writing-review and editing, M.M.N., P.R.-C., C.F.A., P.D.V.; supervision, P.R.-C.; funding acquisition, P.R.-C. All authors have read and agreed to the published version of the manuscript.

Funding: This work was developed within the scope of the project CICECO-Aveiro Institute of Materials, UIDB/50011/2020 \& UIDP/50011/2020, financed by national funds through the FCT/MEC and when appropriate co-financed by FEDER under the PT2020 Partnership Agreement. A.M.A. acknowledges financial support given by POCentro, COMPETE 2020, Portugal 2020, European Community through the FEDER and FCT to Unidade de I\&D Química-Física Molecular of the University of Coimbra (UIDB/00070/2020). FCT is gratefully acknowledged for funding a PhD grant to C.F.A. (SFRH/BD/129040/2017) and a researcher contract to M.M.N. (IF/01468/2015) under the program IF 2015.

Acknowledgments: The STFC Rutherford Appleton Laboratory is thanked for access to neutron beam facilities. CASTEP calculations were made possible due to the computing resources provided by STFC Scientific Computing Department's SCARF cluster.

Conflicts of Interest: The authors declare no conflict of interest.

\section{References}

1. Araujo, C.; Freire, C.S.R.; Nolasco, M.M.; Ribeiro-Claro, P.J.A.; Rudić, S.; Silvestre, A.J.D.; Vaz, P.D. Hydrogen Bond Dynamics of Cellulose through Inelastic Neutron Scattering Spectroscopy. Biomacromolecules 2018, 19, 1305-1313. [CrossRef] [PubMed]

2. Ribeiro-Claro, P.J.A.; Vaz, P.D.; Nolasco, M.M.; Amado, A.M. Understanding the vibrational spectra of crystalline isoniazid: Raman, IR and INS spectroscopy and solid-state DFT study. Spectrochim. Acta Part A Mol. Biomol. Spectrosc. 2018, 204, 452-459. [CrossRef] 
3. Parker, S.F.; Butler, I.R. Synthesis, Computational Studies, Inelastic Neutron Scattering, Infrared and Raman Spectroscopy of Ruthenocene. Eur. J. Inorg. Chem. 2019, 2019, 1142-1146. [CrossRef]

4. Ribeiro-Claro, P.J.; Vaz, P.D.; Nolasco, M.M.; Araujo, C.F.; Gil, F.P.S.C.; Amado, A.M. Vibrational dynamics of 4-fluorobenzaldehyde from periodic DFT calculations. Chem. Phys. Lett. X 2019, 2, 100006. [CrossRef]

5. Bilski, P.; Druzbicki, K.; Jenczyk, J.; Mielcarek, J.; Wasicki, J. Molecular and Vibrational Dynamics in the Cholesterol-Lowering Agent Lovastatin: Solid-State NMR, Inelastic Neutron Scattering, and Periodic DFT Study. J. Phys. Chem. B 2017, 121, 2776-2787. [CrossRef] [PubMed]

6. Sahoo, S.; Ravindran, T.R.; Chandra, S.; Sarguna, R.M.; Das, B.K.; Sairam, T.N.; Sivasubramanian, V.; Thirmal, C.; Murugavel, P. Vibrational spectroscopic and computational studies on diisopropylammonium bromide. Spectrochim. Acta Part A Mol. Biomol. Spectrosc. 2017, 184, 211-219. [CrossRef]

7. Ding, L.; Fan, W.-H.; Chen, X.; Chen, Z.-Y.; Song, C. Terahertz spectroscopy and solid-state density functional theory calculations of structural isomers: Nicotinic acid, isonicotinic acid and 2-picolinic acid. Mod. Phys. Lett. B 2017, 31. [CrossRef]

8. Drużbicki, K.; Mielcarek, J.; Kiwilsza, A.; Toupet, L.; Collet, E.; Pajzderska, A.; Wasicki, J. Computationally Assisted (Solid-State Density Functional Theory) Structural (X-ray) and Vibrational Spectroscopy (FT-IR, FT-RS, TDs-THz) Characterization of the Cardiovascular Drug Lacidipine. Cryst. Growth Des. 2015, 15, 2817-2830. [CrossRef]

9. Bec, K.B.; Grabska, J.; Czarnecki, M.A.; Huck, C.W.; Wojcik, M.J.; Nakajima, T.; Ozaki, Y. IR Spectra of Crystalline Nucleobases: Combination of Periodic Harmonic Calculations with Anharmonic Corrections Based on Finite Models. J. Phys. Chem. B 2019, 123, 10001-10013. [CrossRef]

10. Pawlukojć, A.; Hetmańczyk, Ł. INS, DFT and temperature dependent IR studies on dynamical properties of acetylcholine chloride. Vib. Spectrosc. 2016, 82, 37-43. [CrossRef]

11. Drużbicki, K.; Mikuli, E.; Pałka, N.; Zalewski, S.; Ossowska-Chruściel, M.D. Polymorphism of Resorcinol Explored by Complementary Vibrational Spectroscopy (FT-RS, THz-TDS, INS) and First-Principles Solid-State Computations (Plane-Wave DFT). J. Phys. Chem. B 2015, 119, 1681-1695. [CrossRef] [PubMed]

12. Zhong, L.; Parker, S.F. Structure and vibrational spectroscopy of methanesulfonic acid. R. Soc. Open Sci. 2018, 5. [CrossRef] [PubMed]

13. Deringer, V.L.; George, J.; Dronskowski, R.; Englert, U. Plane-Wave Density Functional Theory Meets Molecular Crystals: Thermal Ellipsoids and Intermolecular Interactions. Acc. Chem. Res. 2017, 50, 1231-1239. [CrossRef] [PubMed]

14. Kresse, G.; Furthmuller, J. Efficiency of ab-initio total energy calculations for metals and semiconductors using a plane-wave basis set. Comput. Mater. Sci. 1996, 6, 15-50. [CrossRef]

15. Dovesi, R.; Erba, A.; Orlando, R.; Zicovich-Wilson, C.M.; Civalleri, B.; Maschio, L.; Rerat, M.; Casassa, S.; Baima, J.; Salustro, S.; et al. Quantum-mechanical condensed matter simulations with CRYSTAL. WILEY Interdiscip. Rev. Mol. Sci. 2018, 8, e1360. [CrossRef]

16. Dovesi, R.; Saunders, V.R.; Roetti, C.; Orlando, R.; Zicovich-Wilson, C.M.; Pascale, F.; Civalleri, B.; Doll, K.; Harrison, N.M.; Bush, I.J.; et al. CRYSTAL17 User's Manual 2017; University of Torino: Torino, Italy, 2017.

17. Clark, S.J.; Segall, M.D.; Pickard, C.J.; Hasnip, P.J.; Probert, M.J.; Refson, K.; Payne, M.C. First principles methods using CASTEP. Zeitschrift Fur Krist. 2005, 220, 567-570. [CrossRef]

18. Pawlukojć, A.; Hołderna-Natkaniec, K.; Bator, G.; Natkaniec, I. L-glutamine: Dynamical properties investigation by means of INS, IR, RAMAN, 1H NMR and DFT techniques. Chem. Phys. 2014, 443, 17-25. [CrossRef]

19. Kearley, G.J. A review of the analysis of molecular vibrations using INS. Nucl. Instruments Methods Phys. Res. Sect. A.. 1995, 354, 53-58. [CrossRef]

20. Verdal, N.; Hudson, B.S. Inelastic neutron scattering and periodic DFT studies of crystalline aromatic materials: Biphenylene-A Mills-Nixon molecule. Chem. Phys. Lett. 2007, 434, 241-244. [CrossRef]

21. Vaz, P.D.; Nolasco, M.; Fonseca, N.; Amado, A.M.; Amorim Da Costa, A.M.; Félix, V.; Drew, M.G.B.; Goodfellow, B.J.; Ribeiro-Claro, P.J.A. C-H. O O hydrogen bonding in 4-phenyl-benzaldehyde: A comprehensive crystallographic, spectroscopic and computational study. Phys. Chem. Chem. Phys. 2005, 7, 3027-3034. [CrossRef]

22. Srishailam, K.; Reddy, B.V.; Rao, G.R. Investigation of torsional potentials, hindered rotation, molecular structure and vibrational properties of some biphenyl carboxaldehydes using spectroscopic techniques and density functional formalism. J. Mol. Struct. 2019, 1196, 139-161. [CrossRef] 
23. Nolasco, M.M.; Amado, A.M.; Ribeiro-Claro, P.J.A. Computationally-assisted approach to the vibrational spectra of molecular crystals: Study of hydrogen-bonding and pseudo-polymorphism. ChemPhysChem 2006, 7, 2150-2161. [CrossRef] [PubMed]

24. Mendes, N.F.C.; Nolasco, M.M.; Ribeiro-Claro, P.J.A. Effects of hydrogen-bond and cooperativity in the vibrational spectra of Luminol. Vib. Spectrosc. 2013, 64, 119-125. [CrossRef]

25. Sardo, M.; Amado, A.M.; Ribeiro-Claro, P.J.A. Hydrogen bonding in nitrofurantoin polymorphs: A computation-assisted spectroscopic study. J. Raman Spectrosc. 2009, 40, 1956-1965. [CrossRef]

26. Groner, P. Barrier, version 22; University of Missouri: Kansas City, MO, USA, 2019.

27. Godunov, I.A.; Bataev, V.A.; Abramenkov, A.V.; Pupyshev, V.I. The Barriers to Internal Rotation of Benzaldehyde and Benzoyl Fluoride: "Reconciliation\{"\} Between Theory and Experiment. J. Phys. Chem. A 2014, 118, 10159-10165. [CrossRef]

28. Araujo, C.F.; Nolasco, M.M.; Ribeiro-Claro, P.J.A.; Rudić, S.; Silvestre, A.J.D.; Vaz, P.D.; Sousa, A.F. Inside PEF: Chain Conformation and Dynamics in Crystalline and Amorphous Domains. Macromolecules 2018, 51, 3515-3526. [CrossRef]

29. Kurapov, P.B.; Klyuev, N.A.; Tyulin, V.I. Potential function of inner rotation of diphenyl. Theor. Exp. Chem. 1988, 24, 190-197. [CrossRef]

30. Tylli, H.; Konschin, H. Raman-spectroscopic study of low frequency vibrations in anisole and anisole-d3. J. Mol. Struct. 1977, 42, 7-12. [CrossRef]

31. Chiang, W.Y.; Laane, J. Fluorescence-spectra and torsional potential functions for trans-stilbene in its $\mathrm{S} 0$ and S1 (pi,pi*) electronic states. J. Chem. Phys. 1994, 100, 8755-8767. [CrossRef]

32. Chiang, W.Y.; Laane, J. Fluorescence-spectra and torsional potential energy functions for 4-methoxy-trans-stilbene in its S0 and S1 (pi,pi*) electronic states. J. Phys. Chem. 1995, 99, 11823-11829. [CrossRef]

33. Vaz, P.D.; Nolasco, M.M.; Gil, F.P.S.C.; Ribeiro-Claro, P.J.A.; Tomkinson, J. Hydrogen-bond dynamics of

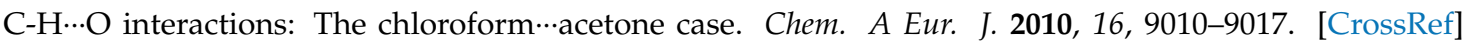
[PubMed]

34. Vaz, P.D.; Nolasco, M.M.; Ribeiro-Claro, P.J.A. Intermolecular C-H center dot center dot center dot O interactions in cyclopentanone: An inelastic neutron scattering study. Chem. Phys. 2013, 427, 117-123. [CrossRef]

35. Li, J.C.; Ross, D.K. Evidence for two kinds of hydrogen bond in ice. Nature 1993, 365, 327-329. [CrossRef]

36. Corsaro, C.; Crupi, V.; Longo, F.; Majolino, D.; Venuti, V.; Wanderlingh, U. Mobility of water in Linde type A synthetic zeolites: An inelastic neutron scattering study. J. Phys. -Condens. Matter 2005, 17, 7925-7934. [CrossRef]

37. Frisch, M.J.; Trucks, G.W.; Schlegel, H.B.; Scuseria, G.E.; Robb, M.A.; Cheeseman, J.R.; Scalmani, G.; Barone, V.; Mennucci, B.; Petersson, G.A.; et al. Gaussian 09, Revision A.02; Gaussian, Inc.: Wallingford, CT, USA, 2009.

38. Refson, K.; Tulip, P.R.; Clark, S.J. Variational density-functional perturbation theory for dielectrics and lattice dynamics. Phys. Rev. B 2006, 73, 155114. [CrossRef]

39. Perdew, J.P.; Burke, K.; Ernzerhof, M. Generalized gradient approximation made simple. Phys. Rev. Lett. 1996, 77, 3865-3868. [CrossRef]

40. Ramirez-Cuesta, A.J. aCLIMAX 4.0.1, The new version of the software for analyzing and interpreting INS spectra. Comput. Phys. Commun. 2004, 157, 226-238. [CrossRef]

41. Jmol: An Open-Source Java Viewer for Chemical Structures in 3D; Jmol Development Team: Minneapolis, MN, USA, 2016; Available online: http://jmol.sourceforge.net/ (accessed on 24 February 2020).

Sample Availability: Samples of the compounds are not available from the authors. 